\title{
Minocycline-Induced Hyperpigmentation in a Patient Treated with Erlotinib for Non-Small Cell Lung Adenocarcinoma
}

\author{
Ann T. Bell ${ }^{a}$ John W. Roman ${ }^{b} \quad$ Max L. Gratrix ${ }^{b} \quad$ Christina E. Brzezniak ${ }^{b}$ \\ ${ }^{a}$ Uniformed Services University of the Health Sciences, Bethesda, MD, USA; ${ }^{b}$ Walter Reed \\ National Military Medical Center, Bethesda, MD, USA
}

\section{Keywords}

Epidermal growth factor receptor inhibitor · Papulopustular eruption · Minocycline · Drug toxicity · Doxycycline

\begin{abstract}
Introduction: While epidermal growth factor receptor (EGFR) inhibitors have improved progression-free survival in patients with non-small cell lung cancer (NSCLC), one of the most common adverse effects is papulopustular skin eruption, which is frequently severe enough to be treated with oral minocycline or doxycycline. Case: We present a case of an 87-yearold man who developed a severe papulopustular skin eruption secondary to erlotinib therapy for NSCLC. Control of the eruption with $100 \mathrm{mg}$ of minocycline twice daily for 8 months eventually led to blue-gray skin hyperpigmentation. After 30 months, this side effect was recognized as minocycline drug deposition, which was confirmed with skin biopsy. Discussion: Compliance with EGFR inhibitor therapy in NSCLC is often challenging due to common side effects, most notably cutaneous skin eruptions. Treatment of cutaneous toxicities is important to preserve patient compliance with targeted cancer therapy. Use of minocycline to treat the most common cutaneous side effect (papulopustular eruption) can in turn cause blue-black skin, eye, or tooth discoloration that can nullify its benefits, resulting in suboptimal patient adherence to cancer therapy. Although this adverse effect is well known in dermatology literature as a risk when using minocycline to treat acne, rosacea, or blistering dis-
\end{abstract}


orders, it is less well documented in oncology literature. We present this case to highlight the need for greater consideration of unique patient characteristics in selecting an oral antibiotic as a treatment modality for EGFR inhibitor skin toxicities.

\section{Introduction}

The use of epidermal growth factor receptor (EGFR) tyrosine kinase inhibitor, erlotinib, as a first- or second-line therapy in EGFR-positive non-small cell lung cancer has increased progression-free survival [1]. The cutaneous side effects of this treatment include papulopustular eruption, xerosis, photosensitivity, alopecia, paronychia, onycholysis, and brittle nails [2]. About $80 \%$ of patients treated with EGFR inhibitors develop a papulopustular eruption and up to $32 \%$ of these are severe enough to be treated with minocycline or doxycycline [2]. Inadequate control of cutaneous side effects leads to impaired quality of life, decreased compliance, and dose reduction [3]. In addition, the tetracyclines used to treat these side effects have their own toxicities. In dermatology literature, minocycline for the treatment of acne, rosacea, and blistering diseases is well known to cause blue-black pigmentation of the skin, eyes, bones, existing scars, and teeth [4]. However, in oncology literature, there are rare reports of minocycline hyperpigmentation when used to treat EGFR inhibitor-induced papulopustular eruptions.

\section{Case Report}

An 87-year-old man with EGFR-positive non-small cell lung adenocarcinoma stage IV (T1aN3M1b) was started on erlotinib $150 \mathrm{mg}$ daily in October 2013. He presented with inflammatory follicular-based papules and pustules over the face after 3 weeks of treatment. Two months later, the eruption involved greater than $50 \%$ of his body and he self-discontinued erlotinib. Subsequently, he was treated with minocycline $100 \mathrm{mg}$ twice daily and was restarted on a decreased dose of erlotinib (50 mg daily). After 8 months of minocycline, he developed new blue-gray patches over his shins that eventually spread to thighs, arms, hands, existing scars, sclera, and teeth (Fig. 1, Fig. 2). For 30 weeks, the discoloration was attributed to erlotinib, although hyperpigmentation is not a common side effect of this therapy. Upon consultation to dermatology, a skin biopsy confirmed dermal pigmentation consistent with minocycline (Fig. 3). His medication was changed to doxycycline and the pigmentation was treated with laser therapy, eventually fading.

\section{Discussion}

Optimization and treatment of skin-related side effects remains paramount for patient adherence to EGFR inhibitor therapy to prevent dose reduction or discontinuation [3]. However, in current oncology literature, there is little distinction between which tetracycline is favored in the treatment of papulopustular eruptions [2]. We present this case to illustrate a common side effect of prolonged minocycline use that is not well reported in oncology literature. 
Pigmentation most commonly occurs on shins, ankles, hands, or forearms, but may involve eyes, bones, existing scars, mucosa, and teeth [5]. Minocycline pigmentation occurs in a dose-dependent fashion, with longer use increasing the risk [4]. Biopsy confirmed this diagnosis with both iron and melanin deposition in macrophages (Fig. 3) [4]. Once recognized, discontinuation of therapy results in eventual fading of pigmentation over months to years. To hasten resolution, Q-switch laser is an effective treatment option [5].

With targeted therapies, progression-free survival in non-small cell lung cancer is extending, leading to greater use of minocycline and greater opportunity for such hyperpigmentation. To protect patient compliance to EGFR inhibitors, we recommend greater consideration of the risks and benefits of each tetracycline class, in combination with patient characteristics, when treating erlotinib-based papulopustular eruption.

\section{Statement of Ethics}

This material has not been published previously, in total or in part, in print or electronic format and is not under consideration by another publication or electronic medium. The patient has given his informed consent. The views expressed in this article are those of the authors and do not reflect the official policy of the Department of Defense or US Government.

\section{Disclosure Statement}

The authors have no conflicts of interest to declare. This research did not receive any specific grant from funding agencies in the public, commercial, or not-for-profit sectors.

\section{References}

1 Greenhalgh J, Dwan K, Boland A, et al: First-line treatment of advanced epidermal growth factor receptor (EGFR) mutation positive non-squamous non-small cell lung cancer. Cochrane Database Syst Rev 2016;(5):CD010383.

2 Lacouture ME, Anadkat MJ, Bensadoun RJ, et al: Clinical practice guidelines for the prevention and treatment of EGFR inhibitor-associated dermatologic toxicities. Support Care Cancer 2011;19:10791095.

-3 Galimon-Collen AFS, Vos LE, Lavrijsen, Ouwerkerk J, Gelderblom H: Classification and management of skin, hair, nail, and mucosal side-effects of epidermal growth factor receptor (EGFR) inhibitors. Eur J Cancer 2007;43:845-851.

4 Eisen D, Hakim MD: Minocycline-induced pigmentation. Incidence, prevention and management. Drug Saf 1998;18:431-440.

-5 Alster TS, Gupta SN: Minocycline-induced hyperpigmentation treated with a 755-nm Q-switched alexandrite laser. Dermatol Surg 2004;30:1201-1204. 


\section{Case Reports in Oncology}
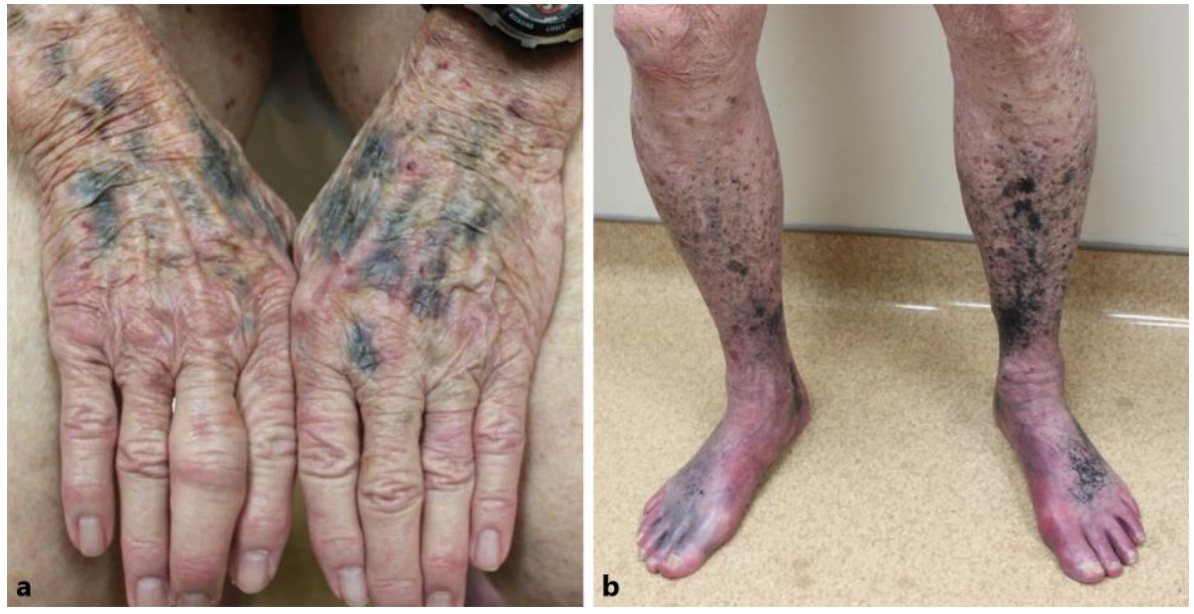

Fig. 1. a Muddy dark blue to black pigmentation on the dorsal hands. $\mathbf{b}$ Black to blue patches on the anterior shins, ankles, and dorsal feet.
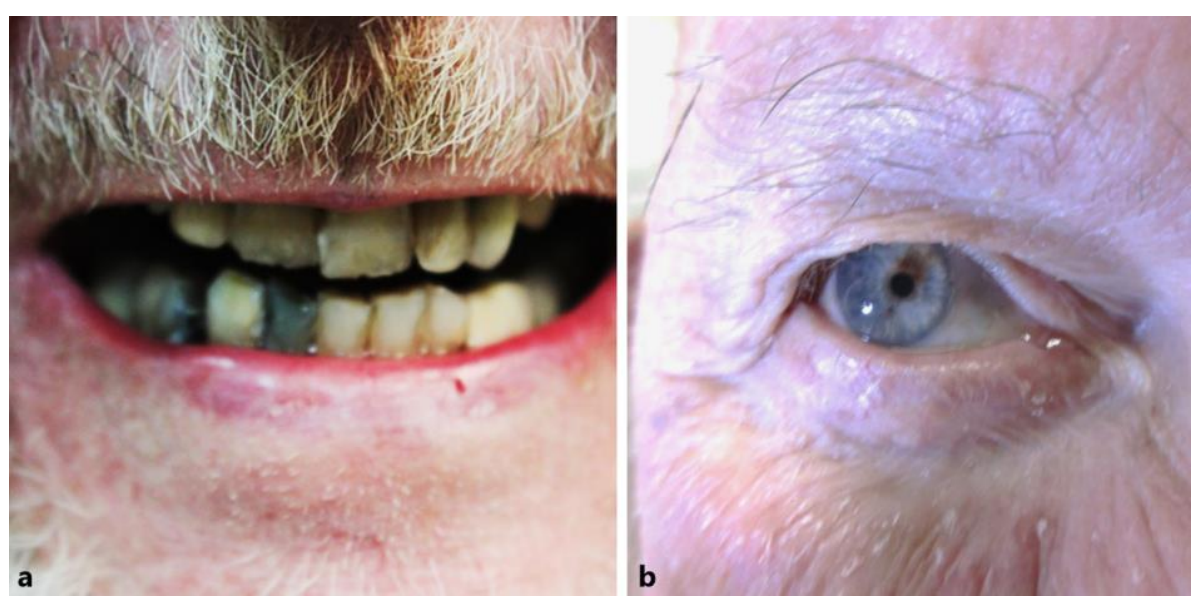

Fig. 2. a Blue-gray discoloration of the inferior teeth most notably of the right lateral incisor. $\mathbf{b}$ A gray discoloration of the medial sclera. 


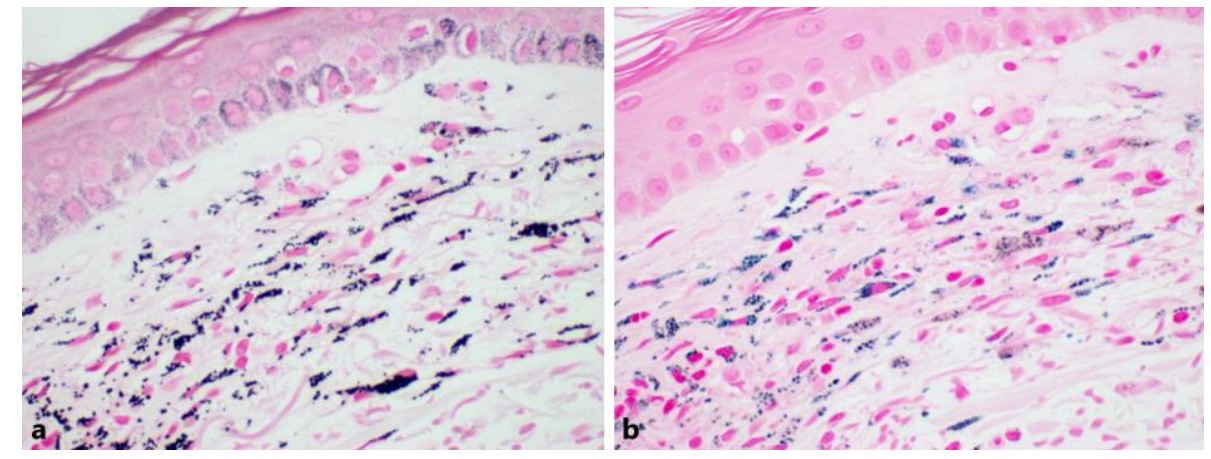

Fig. 3. a Fontana-Masson stain highlighting dermal melanophages engulfing melanin. b Prussian Blue stain showing iron deposition. The combination of dermal pigmentation staining positive for both melanin and iron deposition is consistent with minocycline deposition. Original magnification $\times 400$ ( $\mathbf{a}$ and $\mathbf{b}$ ).

Bell et al.: Minocycline-Induced Hyperpigmentation in a Patient Treated with Erlotinib for Non-Small Cell Lung Adenocarcinoma 